\title{
Pediatric P-ANCA Vasculitis following COVID-19
}

\author{
Yaron Fireizen $^{1}$, Cyrus Shahriary ${ }^{1}$, Maria Imperial ${ }^{1}$, Inderpal Randhawa ${ }^{1}$, Nastasia \\ Nianiaris ${ }^{1}$, and Bugsu Ovunc ${ }^{1}$ \\ ${ }^{1}$ Miller Children's and Women's Hospital Long Beach
}

May 9, 2021

\begin{abstract}
Background: Perinuclear anti-neutrophil cytoplasmic antibodies (P-ANCA), a subset of ANCA, are associated with a multisystem vasculitis affecting small blood vessels in the body. A handful of adult patients who developed vasculitis post-COVID-19 infection have been reported. Although COVID-19 infection has been shown to drive an exaggerated immune response in the pediatric population, such as MIS-C (multisystem inflammatory syndrome in children), only one case of vasculitis following COVID-19 infection has been reported previously in children. Case presentation: Seventeen-year-old male with a past medical history of COVID-19 pneumonia two months prior presented with acute kidney injury/failure and diffuse alveolar hemorrhage (DAH). Rheumatologic workup revealed P-ANCA and Myeloperoxidase (MPO) positivity. Kidney biopsy showed necrotizing glomerulonephritis with limited immune complex deposition. Subsequently, he was treated with pulse steroids, plasmapheresis, and ultimately started on cyclophosphamide. Conclusions: To our knowledge, this report presents the second reported pediatric case of P-ANCA / MPO vasculitis following COVID-19 infection.
\end{abstract}

\section{Pediatric P-ANCA Vasculitis following COVID-19}

Yaron Fireizen, MD*; Cyrus Shahriary, MD*, Maria Elena Imperial, MD*; Inderpal Randhawa, MD*; Nastasia Nianiaris, MD, MS*; Bugsu Ovunc MD, $\mathrm{PhD}^{*}$

Affiliation:

*Pulmonary Division, MemorialCare Miller Children's \& Women's Hospital, Long Beach, CA. University of California, Irvine, School of Medicine, Irvine CA.

Correspondence to:

Yaron Fireizen MD, email \sout: yfireizen@memorialcare.org, Miller Children's \& Women's Hospital Long Beach, 2801 Atlantic Ave, Long Beach, CA 90806. (562) 933-8740

Keywords: COVID-19, Diffuse Alveolar Hemorrhage, P-ANCA Vasculitis

Authors' contributions:

- YF - collected clinical history and data, drafted the initial manuscript, reviewed and revised the manuscript.

- $\mathrm{BO}$ - supervision, reviewed and revised the manuscript.

- NN - collected clinical history and data, reviewed and revised the manuscript.

- SC, IR, EI - reviewed and revised the manuscript.

- All authors approved the final manuscript as submitted and agree to be accountable for all aspects of the work.

Competing interests: The authors declare that they have no competing interests. 
Funding: No grant support given, and no conflicts of financial interest.

Informed Consent: informed consent was obtained from parent and patient. The privacy rights of human subjects were observed.

Availability of data and materials: All data generated or analyzed during this study are included in this published article

Manuscript Words: 864 (without abstract: 663)

Highlights

- Autoimmune development post infectious process.

- Autoimmune disease and its association to COVID-19 infection.

- Pediatric P-ANCA vasculitis post COVID-19.

\section{Abstract}

Background:

Perinuclear anti-neutrophil cytoplasmic antibodies (P-ANCA), a subset of ANCA, are associated with a multisystem vasculitis affecting small blood vessels in the body. A handful of adult patients who developed vasculitis post-COVID-19 infection have been reported. Although COVID-19 infection has been shown to drive an exaggerated immune response in the pediatric population, such as MIS-C (multisystem inflammatory syndrome in children), only one case of vasculitis following COVID-19 infection has been reported previously in children.

Case presentation:

Seventeen-year-old male with a past medical history of COVID-19 pneumonia two months prior presented with acute kidney injury/failure and diffuse alveolar hemorrhage (DAH). Rheumatologic workup revealed P-ANCA and Myeloperoxidase (MPO) positivity. Kidney biopsy showed necrotizing glomerulonephritis with limited immune complex deposition. Subsequently, he was treated with pulse steroids, plasmapheresis, and ultimately started on cyclophosphamide.

Conclusions:

To our knowledge, this report presents the second reported pediatric case of P-ANCA / MPO vasculitis following COVID-19 infection.

\section{Abbreviations}

- Perinuclear anti-neutrophil cytoplasmic antibodies (P-ANCA)

- Coronavirus disease 2019 (COVID-19)

- Myeloperoxidase (MPO)

- Primary medical history (PMH)

- Computed tomography angiography (CTA)

- High Flow Nasal Cannula (HFNC)

- Flexible Fiberoptic Bronchoscopy (FFB)

- Broncho-Alveolar Lavage (BAL)

- Severe Acute Respiratory Syndrome Coronavirus 2 (SARS-CoV-2)

- Polymerase chain reaction (PCR)

- Immunoglobulin G (IgG)

- Acute renal failure (ARF)

Case description:

Seventeen-year-old male with a past medical history $(\mathrm{PMH})$ of obesity and persistent asthma was hospitalized due to COVID-19 pneumonia with hypoxemia, requiring respiratory support via High Flow Nasal Cannula 
(HFNC). He was treated with a 5-day course of Remdesivir, Dexamethasone, Azithromycin, and was placed on Beclomethasone Inhaled $80 \mathrm{mcg}$ ( 2 puffs) twice daily. Moderate bilateral infiltrates on chest x-ray improved upon discharge. One month later, the patient was readmitted with elevated blood pressure, hematuria, proteinuria and diagnosed with acute kidney injury (AKI). AKI was thought to be due to dehydration, with improvement observed after a few days of fluid management and subsequent normalization of blood pressure. He was subsequently discharged.

One month following the second hospital admission, he presented to ER with worsening cough and amber colored urine. He reported fatigue, dry cough, and dyspnea with exertion since initial hospitalization. He also revealed that he had long standing knee, lower back pain, and overall body aches, which were intensified after initial COVID infection. He developed respiratory insufficiency requiring HFNC and was revealed to have anemia (hemoglobin of 5.9). Computed Tomography Angiography (CTA) showed no evidence of pulmonary embolus but did show extensive heterogeneous infiltrates in both lungs with an unusual fluffy central distribution concerning for diffuse alveolar hemorrhage (DAH) (Figure 1). Flexible Fiberoptic Bronchoscopy with Broncho-Alveolar Lavage (FFB/BAL) demonstrated DAH as evidenced by the BAL return color and RBC presence, along with positive hemosiderin-laden macrophages. Respiratory culture and PCR respiratory panel from BAL were negative for an infectious etiology. Rheumatologic evaluation revealed ANA, P-ANCA, and MPO antibody positivity, leading to a diagnosis of ANCA positive vasculitis. Renal biopsy revealed necrotizing glomerulonephritis with limited immune complex deposition.

The patient was initially treated with 3 days of pulse methylprednisolone ( 1 gram per day, divided every 6 hours) and underwent 5 days of plasmapheresis. Steroids were subsequently decreased to $30 \mathrm{mg}$ every 8 hours $(\sim 0.85 \mathrm{mg} / \mathrm{kg} /$ day $)$ of Methylprednisolone, and prior to discharge were transitioned to oral prednisone. Following plasmapheresis, he was started on Cyclophosphamide infusions $10 \mathrm{mg} / \mathrm{kg}$ IV every 2 weeks. His respiratory status improved, acute renal failure resolved, and he was discharged on room air without need for outpatient dialysis.

\section{Discussion}

The precise etiology of autoimmune disorders is yet unclear, though there is growing evidence that there is a role for a multitude of elements, including genetic predisposition and environmental factors ${ }^{1}$. It is proposed that infections with bacterial or viral organisms trigger an exaggerated host immune response via molecular mimicry and activation of pre-primed auto-reactive T-cells and pro-inflammatory mediators ${ }^{1}$. This in turn may lead to tissue damage and multisystem organ dysfunction ${ }^{1}$.

COVID-19 has been shown to share similar pathogenic mechanisms driving an exaggerated immune response $^{2}$. There is a growing evidence to suggest an association between prior COVID-19 infection and ensuing development of autoimmune disorders - primarily in the adult population - such as systemic Lupus Erythematosus, Guillain-Barré syndrome, Kawasaki disease, and Rheumatoid Arthritis ${ }^{2}$. In the pediatric population the most commonly reported autoimmune phenomena associated with COVID-19 infection is Multisystem Inflammatory Syndrome in Children (MIS-C) ${ }^{3}$.

Acute P-ANCA Vasculitis following COVID-19 infection is a rare but documented clinical presentation in the adult population ${ }^{4}$. Only one other pediatric case linking past COVID 19 infection with P-ANCA and MPO positive small vessel vasculitis was reported previously ${ }^{5}$. The other pediatric case also manifested with acute renal failure and DAH. These clinical manifestations are unlikely a direct result of acute COVID-19 infection, as both patients had multiple negative COVID-19 PCR tests while COVID-19 IgG was positive. These multisystem manifestations are likely to be a sequela of COVID-19 infection and its ability to trigger autoimmunity ${ }^{2}$.

\section{Conclusions}

To our knowledge, this report presents the second reported pediatric case of P-ANCA / MPO vasculitis following COVID-19 infection. There is a need for closer and more comprehensive monitoring of patients post COVID-19 infection, particularly of those demonstrating multisystem organ involvement. More research 
is required to better elucidate the link between COVID-19 and its ability to trigger autoimmune disorders, as well as their most appropriate medical management.

References

1. Smatti MK, Cyprian FS, Nasrallah GK, Al Thani AA, Almishal RO, Yassine HM. Viruses and Autoimmunity: A Review on the Potential Interaction and Molecular Mechanisms. Viruses. 2019;11(8):762. Published 2019 Aug 19. doi:10.3390/v11080762

2. Rodríguez Y, Novelli L, Rojas M, et al. Autoinflammatory and autoimmune conditions at the crossroad of COVID-19. J Autoimmun. 2020;114:102506. doi:10.1016/j.jaut.2020.102506

3. Abrams JY, Godfred-Cato SE, Oster ME, et al. Multisystem Inflammatory Syndrome in Children Associated with Severe Acute Respiratory Syndrome Coronavirus 2: A Systematic Review [published online ahead of print, 2020 Aug 5]. J Pediatr. 2020;226:45-54.e1. doi:10.1016/j.jpeds.2020.08.003

4. Hussein A, Al Khalil K, Bawazir YM. Anti-Neutrophilic Cytoplasmic Antibody (ANCA) Vasculitis Presented as Pulmonary Hemorrhage in a Positive COVID-19 Patient: A Case Report. Cureus. 2020;12(8):e9643. Published 2020 Aug 10. doi:10.7759/cureus.9643

5. Powell WT, Campbell JA, Ross F, Peña Jiménez P, Rudzinski ER, Dickerson JA. Acute ANCA Vasculitis and Asymptomatic COVID-19. Pediatrics. 2021 Jan 20:e2020033092. doi: 10.1542/peds.2020-033092. Epub ahead of print. PMID: 33472989.
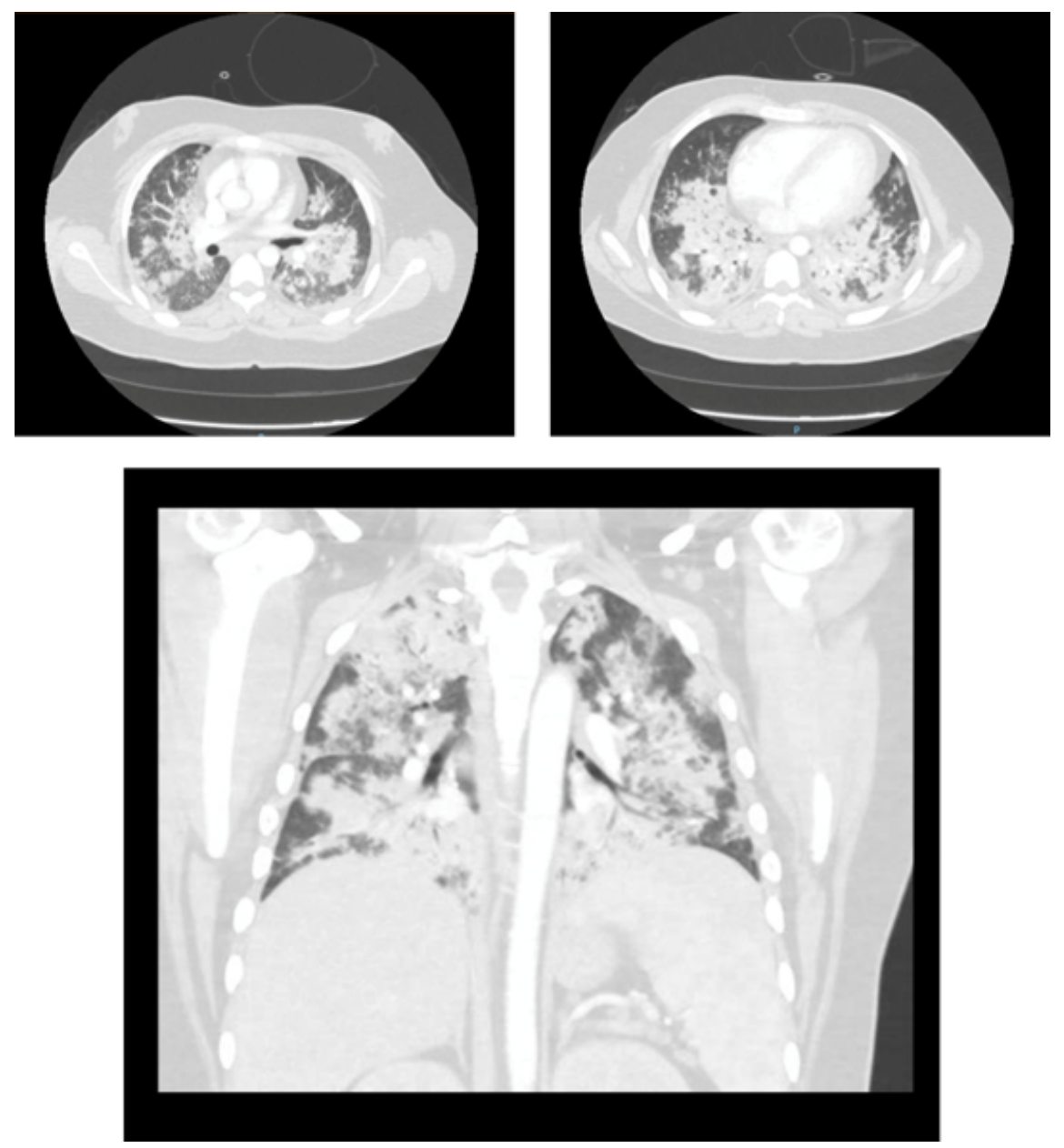